\title{
Depth-averaged flow in presence of submerged cylindrical elements
}

\author{
F. Huthoff \\ Water Engineering and Management, University of Twente, The Netherlands \\ HKV Consultants, Lelystad, the Netherlands \\ D. C. M. Augustijn \& S. J. M. H. Hulscher \\ Water Engineering and Management, University of Twente, The Netherlands
}

\begin{abstract}
The hydraulic resistance of vegetation can play a major role in the hydrodynamics of rivers with extensive natural floodplains. Vegetation penetrates the flow field and thereby causes drag that, in addition to the flow resistance at the bed level, causes energy losses and slows down the flow. Here, these flow processes are studied in an idealized form by treating vegetation as cylindrical roughness elements with homogeneous geometrical dimensions. Based on scaling considerations of the forces involved, depth-averaged flow velocities within the resistance layer and in the free flowing layer above the roughness elements are estimated. This yields a new description of the overall average flow field, which is entirely determined by measurable geometrical boundaries. We tested the new relation against laboratory flume experiments and found very good agreement $\left(\mathrm{R}^{2}=0.98\right)$. The new description even showed realistic results when the depth of flow is of similar size as the height of the roughness elements themselves. This result demonstrates its superiority over commonly used wall-roughness methods.
\end{abstract}

\section{INTRODUCTION}

Submerged vegetation directly influences the discharge capacity in rivers, floodplains or irrigation channels. As a result, the hydraulic resistance of vegetation has been extensively studied in the past decades (for overview see Green 2005). While the hydraulic resistance of solid boundaries with small scale irregularities is quite well understood, situations in which resistance elements are of similar sizes as flow depths still pose large problems (e.g. Smart et al. 2002, Stone and Shen 2002). Especially near the roughness tops, the general form of the most representative velocity profile is still a matter of debate (e.g. Nikora et al. 2004). In case of vegetation, this is further complicated by the natural variability in plant dimensions or streamlining effects.

Laboratory flume studies with natural vegetation have resulted in several empirical flow resistance equations (e.g. Huntington et al. 1992, Copeland 2000). A drawback of these resistance relations is that it is not possible to attribute the relative importance of different flow processes to overall energy losses. General application is therefore difficult, especially in situations that differ much from the original experiments.
More general insight into the hydraulic resistance of vegetation can be achieved by ruling out effects that are due to streamlining effects of leaves and natural variability. Natural vegetation is then replaced by an idealized form: rigid cylindrical stems. Examples hereof can be found in flume studies (e.g. Meijer and van Velzen 1998 or Stone and Shen 2002) and detailed numerical flow models (e.g. Uittenbogaard 2003, Neary 2003). While numerical flow models have shown to represent flow characteristics realistically, the computational effort makes them of limited use for large-scale river applications. Therefore, more simplified solutions are desired that describe the mean flow field in terms of the cylinder characteristics and flow depth.

Stephan and Gutknecht (2002) suggest that the mean deflected plant height is a suitable measure for vegetation resistance. However, Klopstra et al. (1997) claim that a roughness height analogy underestimates vegetative resistance and developed a method that takes into account drag due to individual stems (cylinders) and turbulent mixing. Through depth-integration of the vertical velocity profile an analytical expression for the mean velocity was derived. Unfortunately, the expression is still quite com- 


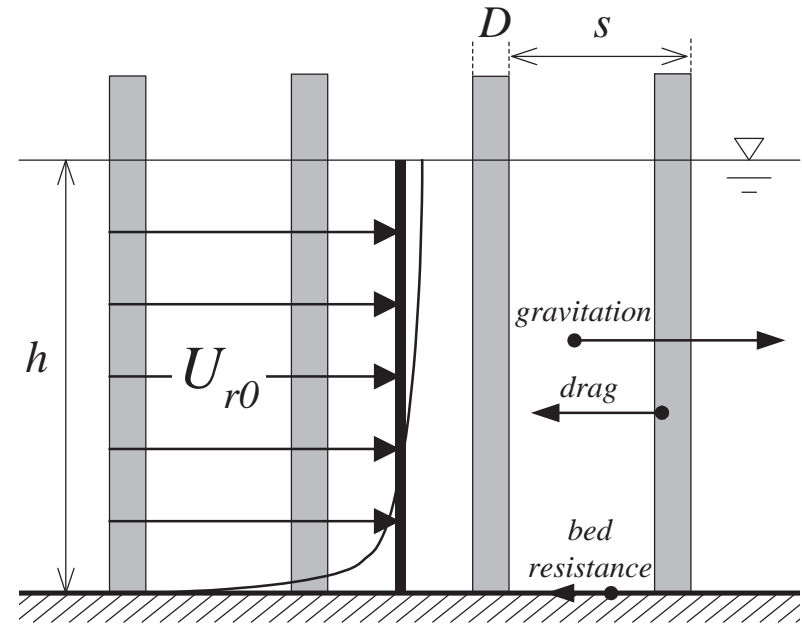

Figure 1 . The depth-averaged velocity $U_{r 0}$ is the result of the balance between the gravitational force (i.e. gravitydriven flow, because of channel with bed slope $i$ ), the drag force and the bed resistance. The individual stems have a diameter $D[\mathrm{~m}]$ and a separation $s[\mathrm{~m}](s=1 / \sqrt{m}, m=$ surface density $\left.\left[\mathrm{m}^{-2}\right]\right)$.

plex and the necessary turbulence closure parameter is poorly understood. Consequently, there are still difficulties in its practical application.

In the current work, flow over vegetation is described by an average-velocity model where distinct flow characteristics are attributed to two separate flow layers. These two regions will be referred to as the surface layer and the resistance layer (see also Figure 2). General scaling assumptions are made, which avoid the necessity of integration over depth and the associated complications of depth-dependence of turbulence intensities. Even though specifics of natural vegetation are neglected, its accurate description of flow through an array of cylindrical stems gives confidence in applications that involve vegetative hydraulic resistance.

\section{FLOW THROUGH VEGETATION}

When a flow field is penetrated by vegetation, turbulent vortices are created in the wake behind the protruding stems (e.g. Akilli and Rockwell 2002). Consequently, in addition to the resistance at the bed, drag effects around the individual plant stems cause the flow to slow down. By replacing the vegetation with a homogeneous field of identical cylindrical stems, the water movement is represented by a bulk force balance (e.g. Baptist 2005):

$$
\underbrace{\rho g h i}_{\text {gravitation }}=\underbrace{\frac{1}{2} \rho C_{D} m D h U_{r 0}^{2}}_{\text {stem drag }}+\underbrace{\rho f U_{r 0}^{2}}_{\text {bed resistance }}
$$

where the individual stems, with diameter $D[\mathrm{~m}]$ and bed surface density $m\left[\mathrm{~m}^{-2}\right]$, exceed the flow depth $h[\mathrm{~m}]$ (see Figure 1). Furthermore, $C_{D}[-]$ is the drag coefficient, $i[-]$ the slope of the channel, and $f[-]$ is the bed resistance function. The separation between individual cylinders $s$ is entirely determined by their bed surface density $m$ :

$$
s=\frac{1}{\sqrt{m}}
$$

Also, the stem diameter $D$ and the stem surface density $m$ can be combined to give a new length scale. Together with the drag coefficient we introduce $b$, the drag length:

$$
b=\frac{1}{C_{D} m D}
$$

By using (3), the average velocity $U_{r 0}[\mathrm{~m} / \mathrm{s}]$ may be written as:

$$
U_{r 0}=U_{r 0}^{*} \sqrt{\frac{1}{1+\frac{2 b}{h} f}}
$$

where $U_{r 0}^{*}$ is the average flow velocity in the resistance layer if no bed resistance effects were present (i.e. when $f=0$ ).

$$
U_{r 0}^{*}=\sqrt{2 b g i}
$$

The average flow velocity $U_{r 0}$ can now be determined if the bed resistance function $f$ is specified. For turbulent flows, several functions for $f$ exist (see e.g. Yen 2002). A commonly used relation for turbulent rough flow was proposed by Strickler (1923), here given in a dimensionally homogeneous form:

$$
\sqrt{f}=\frac{\sqrt{g h i}}{U}=\frac{1}{8}\left(\frac{k_{S}}{h}\right)^{1 / 6}
$$

where Strickler's roughness height $k_{S}[\mathrm{~m}]$ reflects the size of the irregularities on the bed and $U$ the associated depth-averaged velocity. Considering that $k_{S}$ is usually much smaller than the flow depth $h$, the contribution of bed resistance is often negligible in the force balance (1) and, consequently, the average velocity in the resistance layer $U_{r 0}$ is practically equal to $U_{r 0}^{*}$.

Kadlec (1990) warns of the possibility that within the resistance layer flow velocities could reduce to yield a laminar flow field. In that case, the resistance function $f$ is inversely proportional to the Reynolds number (see e.g. Yen 2002).

\section{SUBMERGED VEGETATION}

\subsection{The Resistance Layer}

Whenever cylindrical elements become submerged, the flow in the surface layer has a higher average velocity because in this layer no drag due to the stems is experienced. The energy losses in the surface layer are then entirely due to a shear stress near the top of the resistance layer, which balances the gravitational force that drives the flow. Subsequently, the 


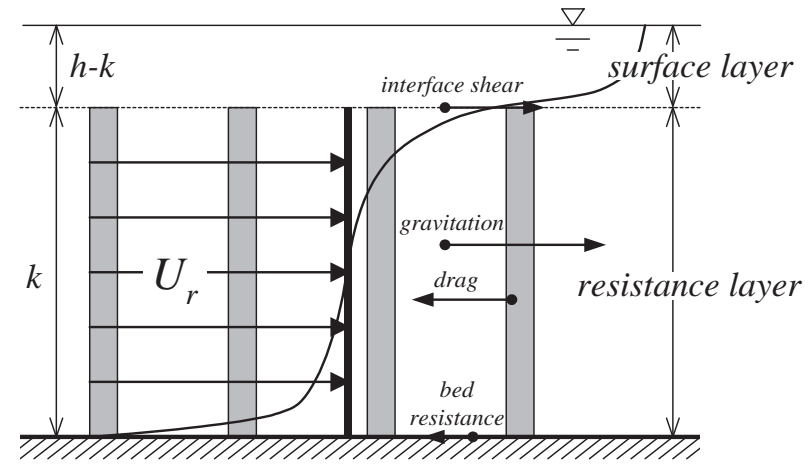

Figure 2. The depth-averaged velocity $U_{r}$ in the layer occupied by the cylindrical stems (i.e. in the resistance layer) in the case that the stems are submerged.

shear stress between surface and resistance layer (i.e. the interface shear) also causes the flow in the top of the resistance layer to speed up (see Figure 2). With an extra component due to the surface layer, the force balance (1) modifies to:

$$
\underbrace{\rho g(h-k) i}_{\text {interface shear }}+\rho g k i=\frac{\rho k U_{r}^{2}}{2 b}+\rho f U_{r}^{2}
$$

The two contributions on the left-hand-side of (7) are due to the gravitational force that acts on the surface and in the resistance layer, respectively. The drag force acts over a depth $k$, as opposed to the total flow depth $h$ in case of (1), where the cylindrical stems were not submerged. As a result, the average velocity in the resistance layer $U_{r}$ becomes:

$$
\frac{U_{r}}{U_{r 0}}=\sqrt{\frac{h}{k}} \quad(h \geq k)
$$

where $U_{r 0}$ is the average velocity in the resistance layer if no free flowing layer above the cylindrical stems were present (equation (4)). Inserting the bed resistance function $f$ as given in (6) into (7), yields for $U_{r 0}$ :

$$
U_{r 0}=U_{r 0}^{*} \sqrt{\frac{1}{1+\frac{b}{32 k}\left(\frac{k_{S}}{h}\right)^{1 / 3}}}
$$

In earlier works, results similar to the square root law in (8) have been found. Smart et al. (2002) point out that with increasing relative roughness, a conceptual drag coefficient model would lead to a squareroot law of the flow resistance. Baptist et al. (2006) used dimensionally aware genetic programming (Keijzer and Babovic 2000) and also found a square root dependence with respect to the relative flow depth $h / k$.

\subsection{The Surface Layer}

Based on physical principles and scaling assumptions, the well-known Strickler equation (6) can be derived

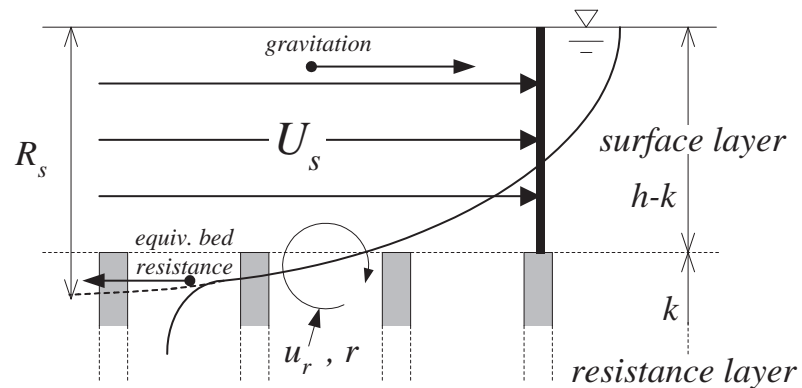

Figure 3. The depth-averaged velocity in the surface layer $U_{s}$. Two hydraulic radii are also shown: $R_{s}$ reflects the depth of the surface layer with respect to an artificial rough bed and $r$ (the spacing hydraulic radius) reflects the roughness height of the artificial rough bed.

for the case of rough channel flow (see Gioia and Bombardelli 2002). The main principles behind the derivation are: (i) a simple force balance; (ii) scaling of turbulent velocity fluctuations to the average flow velocity; and (iii) the concept of a constant energy dissipation rate when large turbulent flow patterns (i.e. eddies) break up into smaller ones (known as Kolmogorov scaling). Here we will follow the same line of reasoning to describe depth-averaged flow over submerged rigid cylinders (i.e. for the surface layer; see Figure 3).

\subsubsection{Shear stress in the surface layer}

In steady flow over a rough bed, the shear stress at the top of the bed $\tau_{k}$ balances the gravitational pull on the water volume above the bed. In analogy, for the surface layer this gives an expression for the shear stress ( $R_{s}$ is the hydraulic radius of the surface layer):

$$
\tau_{k}=\rho g R_{s} i
$$

Furthermore, from the Reynolds averaged NavierStokes equation it follows that the (Reynolds) shear stress at any location in a (2D) flow field is determined by the magnitude of fluctuations in the velocity field (e.g. Pope 2000):

$$
\tau_{x z}=\rho \overline{v_{x} v_{z}}
$$

where $v_{z}$ and $v_{x}$ are velocity fluctuations perpendicular and parallel to the direction of the average flow field. The overbar denotes time-averaging of these fluctuations. Gioia and Bombardelli (2002) argue that for the shear stress near the bed, $v_{x}$ is associated with the characteristic velocity in the surface layer and that the perpendicular component is related to turbulent eddies that dominate between the roughness elements. The average velocity in the surface layer is $U_{s}$ and the velocity associated with the dominating eddies near the artificial rough bed is denoted by $u_{r}$. Following Gioia and Bombardelli the shear stress at the artificial bed (i.e near the top of the resistance layer, see Figure 3) scales as:

$$
\tau_{x z} \propto \rho U_{s} u_{r}
$$


Together with the force balance (10) this yields:

$$
\rho g R_{s} i \propto \rho U_{s} u_{r}
$$

To find a relation for the average velocity in the surface layer $U_{s}$ an independent expression for $u_{r}$ needs to be found. For that purpose, energy considerations of the turbulent flow field are used.

\subsubsection{Turbulent energy and Kolmogorov scaling}

In the Kolmogorov view on turbulent flow, turbulent energy is created through external forcing at the largest scale of the system (energy containing range) and is dissipated to successively smaller scales until eventually viscosity damps the smallest flow patterns (viscous dissipation). The rate of dissipation of turbulent kinetic energy per unit mass is denoted by $\varepsilon$ $\left[\mathrm{m}^{2} / \mathrm{s}^{3}\right]$ and, at large scales, is independent of molecular viscosity. For the energy input at the largest scale (the energy containing scale), a scaling expression for $\varepsilon$ is composed of representative geometrical parameters (hydraulic radius $R_{s}$ ) and the representative flow velocity $\left(U_{s}\right)$, based on dimensional analysis (e.g. Pope 2000).

$$
\varepsilon \propto \frac{U_{s}^{3}}{R_{s}}
$$

Furthermore, if we assume that:

- the turbulence intensity at the artificial rough bed is a result of the energy cascade to smaller scales;

- eddies of size $r$ and velocity $u_{r}$ dominate the flow field near the artificial rough bed;

then the dissipation rate should also scale as:

$$
\varepsilon \propto \frac{u_{r}^{3}}{r}
$$

Now $\varepsilon$ is related to the characteristic velocity $u_{r}$ and the spacing hydraulic radius $r$ that bounds the extent of the dominant local eddies. The characteristic velocity in the resistance layer can be related to the average velocity in the surface layer as (using (14) and (15)):

$$
u_{r} \propto U_{s}\left(\frac{r}{R_{s}}\right)^{1 / 3}
$$

Expression (16) can now be inserted into relation (13). This yields a scaling expression for the average velocity in the surface layer:

$$
U_{s} \propto\left(\frac{R_{s}}{r}\right)^{1 / 6} \sqrt{g R_{s} i}
$$

Note that relation (17) reduces to the Manning/Strickler equation (Manning 1889, Strickler $1923)$ if the total relative flow depth is large $\left(R_{s}\right.$ large $)$ and the spacing hydraulic radius is small ( $r$ becomes an equivalent roughness height). Also, using expression (16) and (17) a scaling equation for the characteristic velocity at the top of the resistance layer $\left(u_{r}\right)$ can be established:

$$
u_{r} \propto\left(\frac{r}{R_{s}}\right)^{1 / 6} \sqrt{g R_{s} i}
$$

To specify the hydraulic radii $R_{s}$ and $r$ in terms of geometrical quantities, we will analyze the two scaling expressions (17) and (18) for their behaviour in certain limiting cases (asymptotic analysis).

\subsubsection{Asymptotic behaviour of hydraulic radii}

Scaling the average velocity in the surface layer $U_{s}$ (17) to the velocity $U_{r 0}(9)$ yields the dimensionless velocity function:

$$
\frac{U_{s}}{U_{r 0}} \propto \sqrt{1+\frac{b}{32 k}\left(\frac{k_{S}}{h}\right)^{1 / 3}} \frac{R_{s}^{2 / 3}}{(2 b)^{1 / 2} r^{1 / 6}}
$$

For most practical cases the bed resistance can be neglected $\left(k_{S}<<h\right)$. Therefore, (19) reduces to:

$$
\frac{U_{s}}{U_{r 0}} \approx \frac{U_{s}}{U_{r 0}^{*}} \propto \frac{R_{s}^{2 / 3}}{(2 b)^{1 / 2} r^{1 / 6}}
$$

We now assume that both $U_{s}$ and $u_{r}$ scale to the relative depth of the surface layer $(h-k) / \ell$, where $\ell$ is an unknown scaling length to be determined empirically. By making $U_{s}$ and $u_{r}$ dimensionless through division with $U_{r 0}$ we define the following power law asymptotics:

$$
\begin{aligned}
\frac{U_{s}}{U_{r 0}} & \propto\left(\frac{h-k}{\ell}\right)^{\gamma} \\
\frac{u_{r}}{U_{r 0}} & \propto\left(\frac{h-k}{\ell}\right)^{\eta}
\end{aligned}
$$

The power law expressions (21) and (22), with constant $\gamma$ and $\eta$, express complete similarity between the considered dimensionless velocities and the relative flow depths of the surface layer (as opposed to incomplete similarity when $\gamma$ and $\eta$ are variable, see Barenblatt 1996). According to (21) and (22) $U_{s}$ and $u_{r}$ allow different rates of convergence, but both eventually vanish when the surface layer disappears. The latter observation immediately limits the validity of the assumptions that lead to (21) and (22): the average flow velocity in the surface layer should always be larger than the flow velocity in the resistance layer $\left(U_{r 0}\right)$, even if the surface layer is extremely shallow. Essentially, power laws (21) and (22) no longer give realistic results when values below unity are found (i.e. when the depth of the surface layer, $h-k$, becomes smaller than $\ell$ ). Nevertheless, we continue with 
(21) and (22), realizing their limitations, and later on evaluate implications for practical application.

Solving for $R_{s}$ and $r$ by combining (21) and (22) with (17) and (18) yields:

$$
\begin{aligned}
& R_{s} \propto 2 b\left(\frac{h-k}{\ell}\right)^{\eta+\gamma} \\
& r \propto 2 b\left(\frac{h-k}{\ell}\right)^{4 \eta-2 \gamma}
\end{aligned}
$$

Next, the two unknowns in the power law $\eta$ and $\gamma$ are determined by investigating the expected behaviour in the case that the water depth becomes much larger than the roughness height. Experimental observations (e.g. Manning 1889, Strickler 1923) have shown that when the depth of flow is much larger than the roughness height, then the flow hydraulic radius can be replaced by the flow depth $h$. For these conditions, the spacing hydraulic radius $r$ as it appears in relation (17) reflects a roughness height and is independent of depth. Thus:

- If $h>>r$ then $R_{s} \sim h$

- If $h>>r$ then $r \sim$ constant

A priori it is not quite clear which parameter, or combination of parameters, $r$ should scale to when $h$ becomes much larger than $k$, but as long as $r$ is not dependent on the depth $h$ then the power law for $r$ in (24) should vanish:

$$
h^{4 \eta-2 \gamma} \sim 1 \Rightarrow 4 \eta-2 \gamma=0
$$

The hydraulic radius in the surface layer scales with $h$; therefore the power law in (23) should behave as:

$$
h^{\eta+\gamma} \sim h \Rightarrow \eta+\gamma=1
$$

Solving for $\eta$ and $\gamma$ by combining expression (25) and (26) yields $\eta=1 / 3$ and $\gamma=2 / 3$. As a result, the power law expressions for $R_{s}$ and $r$ become:

$$
\begin{gathered}
R_{s} \propto 2 b\left(\frac{h-k}{\ell}\right) \\
r \propto 2 b=\frac{2}{m D C_{D}}=\frac{2 s^{2}}{C_{D} D}
\end{gathered}
$$

It was mentioned earlier that $r$ reflects the extent of the largest eddies near the artificial bed. This means that when the separation between roughness elements $s$ is equal to the dynamic diameter of the cylinders (i.e. $C_{D} D$ ) then the largest eddies are bounded by $s$. However, when the diameter of the elements are significantly smaller than the spacing between elements, then (28) shows that $r$ exceeds $s$. In effect, relation
(28) tells us that whenever the spacing between elements is large as compared to the size of the elements themselves, then the eddies are not confined to the space in between the roughness elements.

Expressions (27) and (28) are inserted into the scaling expressions for the average velocity in the surface layer (19), and we arrive at:

$$
\frac{U_{s}}{U_{r 0}} \propto\left(\frac{h-k}{\ell}\right)^{2 / 3}
$$

This is the new scaling expression for the average velocity in the surface layer. Basic assumptions that lead to this result were (i) similarity between velocities and relative flow depths (power laws (21) and (22)) and (ii) correspondence with Manning's equation at large flow depths. A specification of the unknown scaling length $\ell$ in terms of measurable geometric parameters follows from comparison with laboratory experiments.

\section{COMPARISON TO DATA FROM FLUME EX- PERIMENTS}

\subsection{Average velocities in the two flow layers}

The experimental data used in this section is adopted from laboratory flow studies as performed at the $D e$ Voorst WL|Delft Hydraulics facility in 1996 (Meijer and van Velzen 1998, Meijer 1998, also reported in Baptist 2005). Based on four flow experiments without artificial vegetation, the roughness height $k_{S}$ of the flume bed was determined to be $k_{S}=2.3 \pm 0.6$ $\mathrm{mm}$. In the results presented here, this value for $k_{S}$ is used for computing $U_{r 0}$ (equation 9).

Further data from these studies comprise 48 flow experiments with homogeneously distributed cylindrical stems that all have the same diameter $D$ of 8 $\mathrm{mm}$. Flow depths $h$ and cylinder heights $k$ were varied between the experimental runs (three values for $k$ were used: $0.45,0.9$ and 1.5 [m]). Half of the experiments were carried out with a surface density of $m=256$ cylindrical stems per square meter, and the other half with a surface density of $m=64\left[\mathrm{~m}^{-2}\right]$. Flow velocities were measured at different depths $0.10[\mathrm{~m}]$ apart. Using linear interpolation the average flow depth for the surface and the resistance layer were estimated separately. From the measured average velocities in the resistance layer, local Reynolds numbers were calculated $\left(\operatorname{Re} \sim 3 \cdot 10^{4}-4 \cdot 10^{4}\right)$ and, subsequently, the drag coefficients $C_{D}$ for the circular cylinders. Standard works on fluid mechanics report that in such a flow regime the drag coefficient remains fairly constant with a value of nearly 1 (e.g. Schlichting 1979).

Figure 4 shows the measured average velocities in the resistance layer as compared to the predictions of relation (8). Although for slow flows the velocities are 


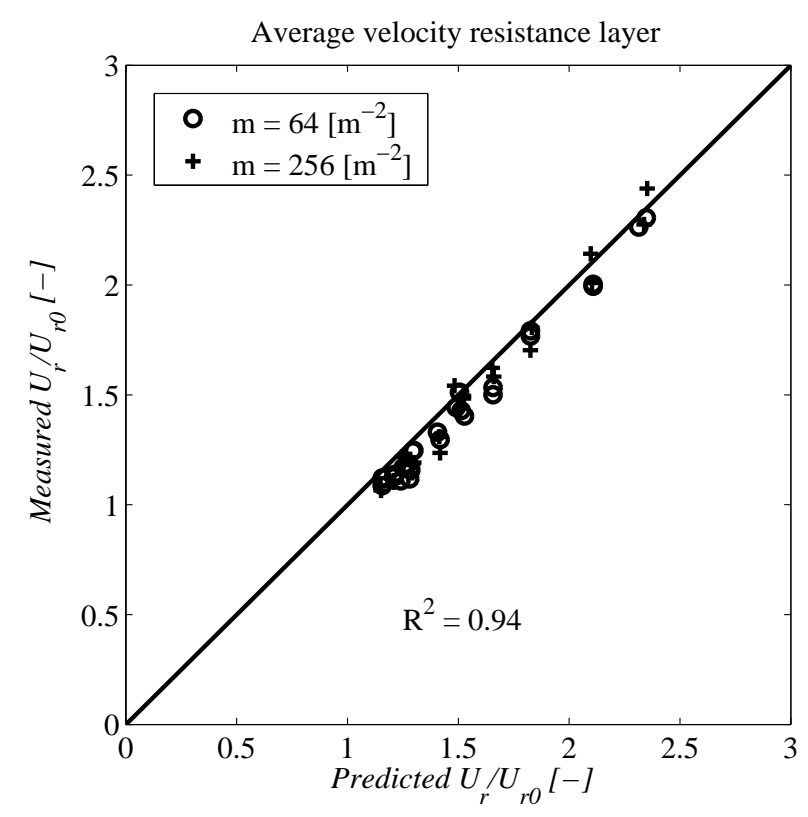

Figure 4. The proposed scaling relation for the depthaveraged velocity $U_{r}$ in the resistance layer (relation (8), on horizontal axis) as compared to results from laboratory flume experiments ( $m=$ number of cylindrical stems per $\mathrm{m}^{-2}$ on the bed surface).

slightly overestimated, by using relation (8) overall agreement with data is very good $\left(\mathrm{R}^{2}=0.94\right)$.

For the specification of the unknown scaling length $\ell$ in relation (29), several combinations of available length scales were attempted $(D, s, b$ and combinations of these). It turns out that for $\ell=s$ the proposed scaling law gives best agreement with laboratory data (see Figure 5). The correlation coefficient is again $\mathrm{R}^{2}=0.94$, in which we have chosen the coefficient of proportionality equal to unity. Apparently, the spacing between the roughness elements is a suitable measure of the extent to which the flow in the surface layer influences the flow field in the resistance layer. Based on this finding, it seems natural to assume that $\ell$ may never exceed the height of the cylinders $k$. By imposing a limit of height $k$ on the scaling length $\ell$, we propose the following scaling expression for the average velocity in the surface layer:

$$
\frac{U_{s}}{U_{r 0}}=\left(\frac{h-k}{\min (s, k)}\right)^{2 / 3}
$$

In section 3.2.3 it was argued that expression (29), or (30), is no longer valid when the flow depth $(h-k)$ is close to or smaller than the scaling length $\ell$ (i.e. $\ell=$ $\min (s, k))$. However, in the considered experimental data the flow depth $(h-k)$ goes down to less than three times $\ell$, and still expression (30) performs well (see Figure 5).

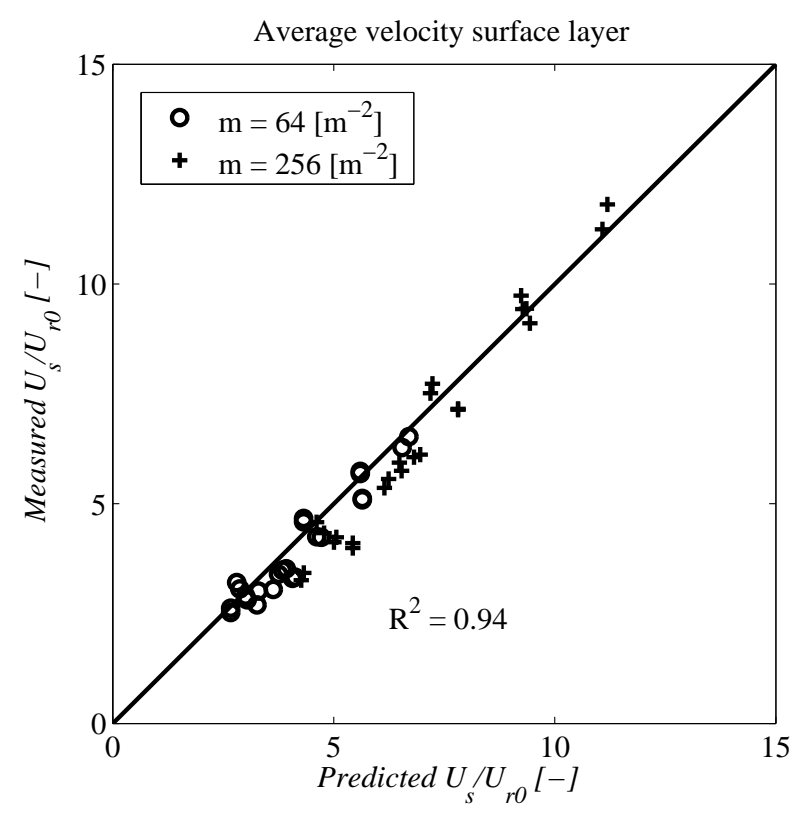

Figure 5. The depth-averaged velocity $U_{s}$ in the surface layer; the proposed relation (30) (on horizontal axis) vs. measured flow velocities.

\subsection{A two-layer scaling model for the entire flow depth}

The average flow velocity for the entire flow depth $U_{T}$ can be found by adding the weighted average flow velocities of the resistance and the surface layer $\left(U_{r}\right.$ and $U_{s}$, respectively):

$$
\frac{U_{T}}{U_{r 0}}=\frac{k}{h} \frac{U_{r}}{U_{r 0}}+\frac{h-k}{h} \frac{U_{s}}{U_{r 0}}
$$

By making use of scaling relations (8) and (30), the expression for the average velocity over the entire flow depth (31) becomes:

$$
\frac{U_{T}}{U_{r 0}}=\sqrt{\frac{k}{h}}+\frac{h-k}{h}\left(\frac{h-k}{\min (s, k)}\right)^{2 / 3}
$$

In the previous section the validity of (30) was addressed for cases when the depth of the surface layer $(h-k)$ becomes small. Significant errors in (30) are expected when $(h-k)$ becomes close to or smaller than $\ell$. However, relation (31) shows that in such cases the average velocity in the surface layer $\left(U_{s}\right)$ only contributes little to the overall average velocity $\left(U_{T}\right)$. Therefore, errors in (30) due to shallowness of the surface layer only have a minor impact on the total depth-averaged velocity $U_{T}$.

In Figure 6, the predictions of relation (32) are shown in comparison to the measured average flow velocities (interpolation between flow velocities measured at different depths). The combined performance of the velocity scaling expressions (8) and (30) is even better than found before, yielding a correlation coefficient of $\mathrm{R}^{2}=0.98$. 


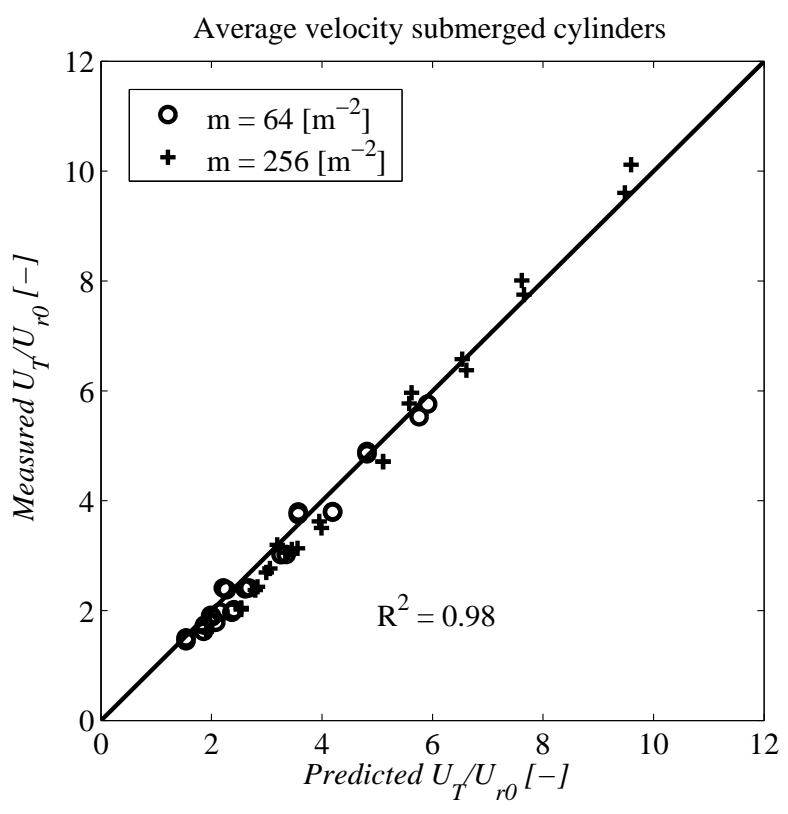

Figure 6 . The depth-averaged velocity $U_{T}$ over the entire flow depth (relation (32), on horizontal axis) vs. measured average velocities.

\section{CONCLUSIONS}

For open channel flow over rough surfaces, several suitable relations exist that relate the average flow velocity to a roughness height that reflects the resistance of the bed. However, when the bed is covered with large roughness elements such as vegetation, or cylindrical elements, these methods are theoretically no longer valid. In this paper, a two-layer scaling model is proposed that treats the flow field above and in between the roughness elements separately, and, when combined, gives a description of the depth-averaged velocity of the entire flow field. When comparing the newly derived description to results from flume experiments, very good agreement is found (correlation coefficient $\mathrm{R}^{2}=0.98$ ). The scaling length $\ell$, that completes the velocity relation in the surface layer, is found to be well represented by the spacing between the cylindrical stems. It is expected that this is no longer the case when the height of the cylinders is smaller than the distance between them. Further investigations with different geometrical properties of the cylinders, and different spatial distributions, should be carried out to substantiate this claim.

The difficulty of describing flow over vegetation is primarily due to flow characteristics near the top of the vegetation, as reflected in the scaling length $\ell$ that is necessary to describe the flow in the surface layer. The flow field in between the resistance elements is satisfactorily described by drag forces. However, for the surface layer energy losses are due to the turbulent patterns, for which in a bulk flow description a representative closure parameter is required. In the used experimental data set, vegetation was represented by homogeneously distributed identical rigid stems, and the turbulent length scale was adequately described by one geometrical length scale from within the resistance layer. Future work will be focussed on the characteristics of such a scaling length when geometric variability between the resistance elements exist. Eventually, the aim is to describe flow over natural vegetation, which involves further complications as stem flexibility, or leaves and side branches that individual plants may have.

\section{NOTATION}

\begin{tabular}{|c|c|c|}
\hline$\rho$ & {$\left[\mathrm{kg} / \mathrm{m}^{3}\right]$} & Water density \\
\hline & {$\left[\mathrm{m} / \mathrm{s}^{2}\right]$} & Gravitational acceleration \\
\hline & {$[-]$} & Bed slope of channel \\
\hline$h$ & [m] & Flow depth \\
\hline$k$ & {$[\mathrm{~m}]$} & Height of cylinders \\
\hline$C_{D}$ & {$[-]$} & Drag coefficient \\
\hline$m$ & {$\left[\mathrm{~m}^{-2}\right]$} & Bed surface density of cylinders \\
\hline$D$ & {$[\mathrm{~m}]$} & Diameter of cylinders \\
\hline$s$ & [m] & Separation between cylinders \\
\hline$b$ & [m] & Drag length \\
\hline$f$ & {$[-]$} & Bed resistance function \\
\hline$k_{S}$ & [m] & Strickler's roughness height \\
\hline$U_{r}$ & {$[\mathrm{~m} / \mathrm{s}]$} & $\begin{array}{l}\text { Depth-averaged flow velocity in } \\
\text { resistance layer (general) }\end{array}$ \\
\hline$U_{r 0}$ & {$[\mathrm{~m} / \mathrm{s}]$} & As $U_{r}$, for $h<k$ \\
\hline$U_{r 0}^{*}$ & {$[\mathrm{~m} / \mathrm{s}]$} & As $U_{r 0}$, neglecting bed resistance \\
\hline$U_{s}$ & {$[\mathrm{~m} / \mathrm{s}]$} & $\begin{array}{l}\text { Depth-averaged flow velocity in } \\
\text { surface layer }\end{array}$ \\
\hline$U_{T}$ & {$[\mathrm{~m} / \mathrm{s}]$} & $\begin{array}{l}\text { Depth-averaged flow velocity over } \\
\text { total flow depth }\end{array}$ \\
\hline$u_{r}$ & {$[\mathrm{~m} / \mathrm{s}]$} & $\begin{array}{l}\text { Characteristic eddy-velocity near } \\
\text { top of resistance elements }\end{array}$ \\
\hline$R_{s}$ & {$[\mathrm{~m}]$} & Hydraulic radius of surface layer \\
\hline$r$ & [m] & Spacing hydraulic radius \\
\hline 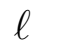 & [m] & Unknown scaling length \\
\hline$\varepsilon$ & {$\left[\mathrm{m}^{2} / \mathrm{s}^{3}\right]$} & Energy dissipation rate \\
\hline & {$[\mathrm{m} / \mathrm{s}]$} & Velocity fluctuation \\
\hline
\end{tabular}

\section{ACKNOWLEDGEMENTS}

This research is supported by the Technology Foundation STW, applied science division of NWO and the technology programme of the Ministry of Economic Affairs (the Netherlands). Earlier stages of this research were carried out with the support of the German Federal Institute of Hydrology (Bundesanstallt für Gewässerkunde). Furthermore, we gratefully thank Francisco Fontanele Araujo from the Department of Applied Physics at the University of Twente for his critical views and comments.

\section{REFERENCES}

Akilli, H. and D. Rockwell (2002). Vortex formation from a cylinder in shallow water. Physics of Fluids 14(9), 2957-2967. 
Baptist, M. J. (2005). Modelling Floodplain Biogeomorphology. Ph. D. thesis, Delft University of Technology.

Baptist, M. J., V. Babovic, J. Rodriguez Uthurburu, M. Keijzer, R. E. Uittenbogaard, A. Mynett, and A. Verwey (2006). On inducing equations for vegetation resistance. Journal of Hydraulic Research. (subm.).

Barenblatt, G. I. (1996). Scaling, self-similarity, and intermediate asymptotics, Volume 14 of Cambridge Texts in Applied Mathematics. Cambridge University press.

Copeland, R. R. (2000). Determination of flow resistance coefficients due to shrubs and woody vegetation. Technical report, U.S. Army Engineer Research and Development Center, Vicksburg, MS, USA.

Gioia, G. and F. A. Bombardelli (2002). Scaling and similarity in rough channel flows. Physical Review Letters 88(1), 14501-14504.

Green, J. C. (2005). Modelling flow resistance in vegetated streams: review and development of new theory. Hydrological Processes 19, 1245-1259.

Huntington, S. W., E. Whitehead, P. Brown, and P. Hollindrake (1992). The hydraulic roughness of vegetated channels. Technical Report SR 305, HR Wallingford, Wallingford, England.

Kadlec, R. H. (1990). Overland flow in wetlands: vegetation resistance. Journal of Hydraulic Engineering 116(5), 691-706.

Keijzer, M. and V. Babovic (2000). Genetic programming within a framework of computer-aided discovery of scientific knowledge. In D. Whitley, D. Goldberg, E. Cantu Paz, L. Spectro, I. Parmee, and H. G. Beyer (Eds.), Proceedings of the Genetic and Evolutionary Computation Conference, Las Vegas, NV, USA. Morgan Kaufman.

Klopstra, D., H. J. Barneveld, J. M. van Noortwijk, and E. H. van Velzen (1997). Analytical model for hydraulic roughness of submerged vegetation. In Managing Water: Coping with scarcity and abundance, Proceedings of the 27th IAHR Congress, San Fransisco, CA, USA, pp. 775-780.

Manning, R. (1889). On the flow of water in open channels and pipes. Transactions of the Institution of Civil Engineers of Ireland 20, 161-207.

Meijer, D. G. (1998). Modelproeven overstroomde vegetatie. Technical Report PR121, HKV Consultants, Lelystad, the Netherlands.

Meijer, D. G. and E. H. van Velzen (1998). Prototypescale flume experiments on hydraulic roughness of submerged vegetation. Technical report, HKV Consultants, Lelystad, The Netherlands.

Neary, V. S. (2003). Numerical solution of fullydeveloped flow with vegetative resistance. Journal of Engineering Mechanics 129(5), 558-563.
Nikora, V., K. Koll, I. McEwan, S. McLean, and A. Dittrich (2004). Velocity distribution in the roughness layer of rough bed flows. Journal of Hydraulic Engineering 130(1), 1036-1042.

Pope, S. B. (2000). Turbulent Flows (third ed.). Cambridge University press.

Schlichting, H. (1979). Boundary Layer Theory (7th ed.). New York: McGraw-Hill.

Smart, G. M., M. J. Duncan, and J. M. Walsh (2002). Relatively rough flow resistance equations. Journal of Hydraulic Engineering 128(6), 568-578.

Stephan, U. and D. Gutknecht (2002). Hydraulic resistance of submerged flexible vegetation. Journal of Hydrology 269, 27-43.

Stone, B. M. and H. T. Shen (2002). Hydraulic resistance of flow in channels with cylindrical roughness. Journal of Hydraulic Engineering 128(5), 500-506.

Strickler, A. (1923). Beitrage zur Frage der Geschwindigkeitsformel und der Rauhigkeitszahlen für Ströme, Kanäle und geschlossene Leitungen. Mitteilungen des Amtes für Wasserwirtschaft 16, Eidg. Department des Innern, Bern, Switserland.

Uittenbogaard, R. (2003). Modelling turbulence in vegetated aquatic flows. In International workshop on riparian forest vegetated channels: hydraulic, morphological and ecological aspects, Trento, Italy.

Yen, B. C. (2002). Open channel flow resistance. Journal of Hydraulic Engineering 128(1), 20-39. 